\title{
Leishmania RNA Virus 1 (LRV-1) in Leishmania (Viannia) braziliensis Isolates from Peru: A Description of Demographic and Clinical Correlates
}

\author{
Ruwandi Kariyawasam, ${ }^{1}$ Rachel Lau, ${ }^{2}$ Braulio M. Valencia, ${ }^{3}$ Alejandro Llanos-Cuentas, ${ }^{4}$ and Andrea K. Boggild ${ }^{1,2,5,6 *}$ \\ ${ }^{1}$ Institute of Medical Sciences, University of Toronto, Toronto, Canada; ${ }^{2}$ Public Health Ontario Laboratory, Toronto, Canada; ${ }^{3}$ Viral Immunology \\ Systems Program, Kirby Institute, University of New South Wales, Australia; 'Instituto de Medicina Tropical "Alejandro von Humboldt", Lima, Peru; \\ ${ }^{5}$ Department of Medicine, University of Toronto, Toronto, Canada; ${ }^{6}$ Tropical Disease Unit, Toronto General Hospital, Toronto, Canada
}

\begin{abstract}
RNA virus 1-1 (LRV-1-1) is a dsRNA virus identified in isolates of Leishmania (Viannia) braziliensis and thought to advance localized cutaneous leishmaniasis (LCL) to mucocutaneous or mucosal leishmaniasis (MCL/ML). We examined the prevalence of LRV-1 and its correlation to phenotypes of American tegumentary leishmaniasis caused by $L$. $(V$.$) braziliensis from Peru to better understand its epidemiology. Clinical isolates of L$. $(V$.) braziliensis were screened for LRV-1 by real-time polymerase chain reaction (PCR) and stratified according to the phenotype: LCL ( $<4$ ulcers in number) MCL/ML; inflammatory ulcers (erythematous, purulent, painful ulcers with or without lymphatic involvement) or multifocal ulcers ( $\geq 4$ in $\geq 2$ anatomic sites). Proportionate LRV-1 positivity was compared across phenotypes. Of $78 \mathrm{~L}$. (V.) braziliensis isolates, 26 (54.2\%) had an inflammatory phenotype, 22 (28\%) had the MCL/ML phenotype, whereas 30 (38.5\%) had LCL. Mucocutaneous or mucosal leishmaniasis was found exclusively in adult male enrollees. Leishmania RNA virus 1 positivity by phenotype was as follows: $9 / 22$ (41\%) with MCL/ML; $5 / 26(19 \%)$ with an inflammatory/multifocal cutaneous leishmaniasis phenotype; and $7 / 30(23 \%)$ with LCL $(P=0.19)$. Leishmania RNA virus 1 positivity was not associated with age $(P=0.55)$ or gender $(P=0.49)$. Relative LRV-1 copy number was greater in those with $\mathrm{MCL} / \mathrm{ML}$ than those with inflammatory/multifocal CL $(P=0.02)$. A direct association between LRV-1 status and clinical phenotype was not demonstrated; however, relative LRV-1 copy number was highest in those with MCL/ML. Future analyses to understand the relationship between viral burden and pathogenesis are required to determine if LRV-1 is truly a contributor to the MCL/ML phenotype.
\end{abstract}

\section{INTRODUCTION}

American tegumentary leishmaniasis (ATL) includes cutaneous leishmaniasis (CL), mucocutaneous (MCL), and mucosal leishmaniasis (ML), affecting one to two million people in the Americas. ${ }^{1}$ Localized CL (LCL) is generally a self-healing disease characterized by ulcerative, nodular, or verrucous lesions on the skin caused by several Leishmania spp. and endemic to many parts of the world, including Peru. ${ }^{1-2}$ Other clinical manifestations of $C L$ include inflammatory $C L$ where ulcers are associated with erythema, purulent exudate, pain and/or lymphatic involvement, and more recently, atypical $\mathrm{CL}$, which has been documented in an endemic region of Brazil. ${ }^{3}$ To add, other forms include diffuse $\mathrm{CL}$ with multiple non-ulcerative nodules, ${ }^{1}$ and disseminated leishmaniasis, defined as maculopapular lesions identified in two or more anatomical sites ranging from 10 to 300 in number. ${ }^{3}$ Mucosal leishmaniasis is a form of the disease affecting mucous membranes such as the nose, mouth, pharynx, and larynx, more often attributed to sequela of the initial $C L$ infection in Latin America, whereas MCL involves both cutaneous and mucosal lesions. ${ }^{1}$ This diverse phenotypology reflects a complex relationship between host, parasite, and vector factors ${ }^{1}$ (extensively reviewed in ref. 1), with strong geographic- and species-specific preponderances to cutaneous manifestations of disease.

To add to this complexity of ATL pathogenesis, the presence of a double-stranded RNA virus, Leishmania RNA virus 1 (LRV-1), has been identified in up to a quarter of certain strains of Leishmania (Viannia) spp., including Leishmania (Viannia) braziliensis and Leishmania (Viannia) guyanensis. Leishmania RNA virus 1 found in New World Viannia strains are identified as LRV-1, with 14 subtypes (LRV-1-1-LRV-1-14) predominantly

* Address correspondence to Andrea K. Boggild, Tropical Disease Unit, Toronto General Hospital, 200 Elizabeth St., 13EN-218, Toronto M5G 2C4, Canada. E-mail: andrea.boggild@utoronto.ca found in the Amazon basin. ${ }^{4,5}$ Genetic diversity between LRV-1 and parasite species exists; however, the viruses from the same parasite species have shown less heterogeneity. ${ }^{6}$ It is hypothesized that the presence of LRV- 1 will advance $10-15 \%$ of $C L$ to MCL/ML stemming from an overactive immune response leading to severe immunopathological tissue infiltration and destruction. ${ }^{7-11}$

LRV-1 has been documented in $20-25 \%$ of clinical isolates of $L$. (V.) guyanensis and L. (V.) braziliensis found in Brazil and Peru and has been associated with first-line treatment failure. ${ }^{7,12}$ Studies have also indicated higher levels of LRV-1 in metastasizing versus non-metastasizing strains of $L$. (V.) guyanensis, which were correlated with increased levels of proinflammatory cytokines and chemokines, including tumor necrosis factor (TNF- $\alpha$ ), interleukin 6 (IL-6), interferon-gamma inducible protein 10 (CXCL10), chemokine ligand 4 (CCL4), and chemokine ligand 5 (CCL5) after recognition by toll-like receptor 3 in human and murine studies. ${ }^{7}$ On the other hand, in a human macrophage model, we have documented that LRV-1 in $L$. (V.) braziliensis was correlated with lower expression levels of TNF- $\alpha$, IL- $6, I L-1 \beta$, and CXCL10 and increases in superoxide dismutase. ${ }^{11}$ Given that LRV-1 may predict and correlate with more severe clinical manifestations of ATL and given that Peru is one of the top worldwide contributors of $\mathrm{CL}, \mathrm{ML}$, and $\mathrm{MCL}$, we aimed to understand its prevalence in clinical isolates of $L$. $(V$.) braziliensis and the possible epidemiologic association between different clinical phenotypes of ATL from Peru.

\section{MATERIALS AND METHODS}

Ethics approval. Approval for this study was obtained from the Ethics Review Board of Public Health Ontario, the Research Ethics Board of University of Toronto, and the Institutional Review Board of Hospital Nacional Cayetano Heredia, Lima, Peru. 
Specimen enrollment. Unique surplus discard clinical specimens of Leishmania spp. were identified from Public Health Ontario Laboratory and the Leishmania Clinic of the Instituto de Medicina Tropical Alexander von Humboldt, Lima, Peru, between 2012 and 2018 (Supplemental Table 1, Figure 1). Biobanked isolates were confirmed as Leishmania spp. by multiplex real-time PCR targeting Leishmania $18 \mathrm{~S}$ rRNA, following clinical testing, which included microscopic examination of Giemsa-stained smears and/or culture by certified medical laboratory technologists.

Clinical data. De-identified clinical data of source patients collected from test requisitions and case record forms were stratified into the following phenotypes: MCL/ML (simultaneous cutaneous infection and/or destruction of the mucosa), inflammatory ulcers (ulcers with associated erythema, purulent exudate, and pain with or without lymphatic involvement), or multifocal/disseminated ulcers (ulcers in $\geq 2$ anatomic sites and $\geq 4$ in number) as per the Infectious Diseases Society of America guidelines, ${ }^{2}$ understanding that the pathogenesis underpinning mucosal versus severe cutaneous manifestations of Leishmania infection are quite different. Localized cutaneous leishmaniasis was defined as of $<4$ ulcers in number. ${ }^{2}$

DNA extraction. DNA extraction was performed using the Qiagen DNA Mini Kit (Qiagen, Germantown, MD) using $200 \mu \mathrm{L}$ of cultured specimen with a final elution volume of $60 \mu \mathrm{L}$. In the case of primary clinical specimens, including filter paper lesion impressions (FPLIs) (ThermoFisher, Waltham, MA), biopsies, and cytology brushes (VWR, Radnor, PA), specimens were soaked in $200 \mu \mathrm{L}$ of TE (ThermoFisher Scientific) before extraction to achieve sufficient volume and DNA concentration and eluted in $60 \mu \mathrm{L}$ nuclease-free water (ThermoFisher Scientific).

RNA extraction. RNA was extracted from cultured promastigotes using the Cells Protocol of the QIAamp RNA Mini Kit (Qiagen) and eluted with $50 \mu \mathrm{L}$ of RNase-free water (ThermoFisher Scientific). RNA was extracted from tissue biopsy and cytology brushes using the Fibrous Tissue Protocol

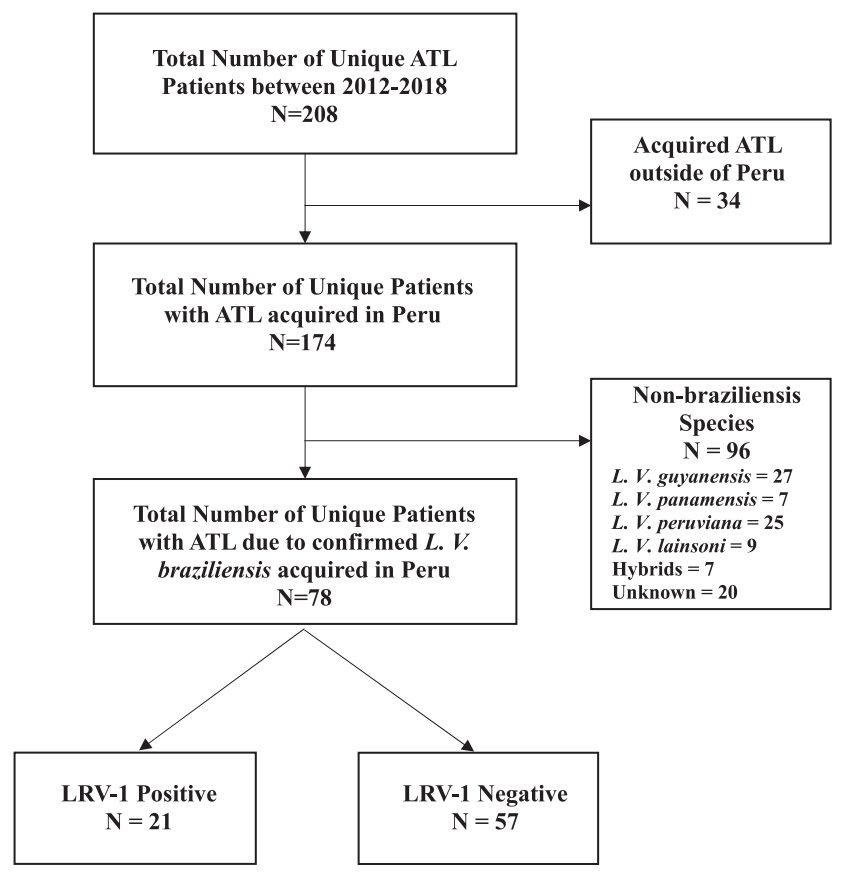

FIGURE 1. Workflow of sample identification and stratification of patients with confirmed Leishmania (Viannia) braziliensis. from the Qiagen RNeasy Micro Kit (Qiagen) with the addition of carrier RNA (Qiagen) and eluted with $14 \mu \mathrm{L}$ RNase-free water (ThermoFisher Scientific). RNA was extracted from FPLIs with the QIAmp RNA Blood Mini Kit (Qiagen) and eluted with $30 \mu \mathrm{L}$ RNase-free water. An in-column DNase treatment was included using the Qiagen rDNase Set (Qiagen) as per the manufacturer's protocol.

cDNA synthesis and purification. cDNA was performed using $10 \mu \mathrm{L}$ of RNA in combination with the Superscript II Reverse Transcriptase and random hexamers (ThermoFisher Scientific). ${ }^{11}$ PCR purification was performed using the Qiagen QIAquick PCR Purification Kit (Qiagen) and eluted with 60 $\mu \mathrm{L}$ nuclease-free water (ThermoFisher Scientific).

Species identification. Species identification was performed using the following gene targets by end-point PCR: internal transcriber space 1 (ITS1), ITS2, cysteine proteinase $\mathrm{B}$, heat shock protein 70 , mannose phosphate isomerase, zinc-dependent metalloproteinase (GP63), and confirmatory Sanger sequencing. ${ }^{11,13-15}$ Restriction fragment length polymorphism analysis was performed on each product of endpoint PCR. ${ }^{11,14-15}$

Sanger sequencing. Sanger sequencing was performed using $1 \mu \mathrm{L}$ of PCR product, $2 \mu \mathrm{L}$ of BigDye, $3 \mu \mathrm{L}$ of buffer, and $2 \mu \mathrm{L}$ of $10 \mu \mathrm{M}$ of primer (ThermoFisher Scientific). ${ }^{11}$ The following cycling conditions were used on the Veriti $\mathrm{ABI}$ Thermal Cycler (Applied Biosystems, Waltham, MA): 1 minute at $96^{\circ} \mathrm{C}$, 25 cycles of 10 seconds at $96^{\circ} \mathrm{C}, 5$ seconds at $50^{\circ} \mathrm{C}$, and 4 minutes at $60^{\circ} \mathrm{C}$. The product was cleaned using $45 \mu \mathrm{L}$ of SAM Solution and $10 \mu \mathrm{L}$ of beads set on a shaking incubator for 30 minutes. ${ }^{11}$ The products were then centrifuged for 2 minutes at 2,000 $\mathrm{g}$ before being loaded onto the Applied Biosystems 3730xI DNA Analyzer (Applied Biosystems). Data were standardized using the Sequencing Analyzer program and Basic Local Alignment Search Tool (BLAST) search engine was used to analyze the sequence. ${ }^{11}$

Leishmania RNA virus 1 detection and quantification. Leishmania RNA virus 1 was detected in isolates of $L$. $(V$.) braziliensis by real time PCR using two primer sets, set $A$ and set $B$, respectively (Figure 1). ${ }^{11,16-17}$ Leishmania kinetoplastid membrane protein 11 (kmp11) was used as a reference for quantification where sufficient RNA volume for quantification permitted this analysis. ${ }^{11,18}$ A SYBR Green assay was set up using $1 \times$ SYBR Select Master Mix, $250 \mathrm{nM}$ final concentration of forward and reverse primers, and $5 \mu \mathrm{L}$ of cDNA in a total volume of $20 \mu \mathrm{L} .{ }^{11}$ The ABI $7900 \mathrm{HT}$ real time instrument was set to the following conditions: uracil-DNA glycosylase (UDG) activation at $50^{\circ} \mathrm{C}$ for 2 minutes, polymerase activation at $95^{\circ} \mathrm{C}$ for 2 minutes, followed by 45 cycles of $95^{\circ} \mathrm{C}$ for 15 seconds, and $60^{\circ} \mathrm{C}$ for 1 minute. ${ }^{11} \mathrm{~A}$ dissociation step of $95^{\circ} \mathrm{C}$ for 15 seconds, $60^{\circ} \mathrm{C}$ for 15 seconds, and another $95^{\circ} \mathrm{C}$ for 15 seconds was added at the end to generate a melting curve to check for specificity of amplification. Each isolate was run in triplicate and contained the L. (V.) guyanensis American Type Culture Collection $^{\circledR}\left(\right.$ ATCC $\left.^{\circledR}\right)$ 50126 ${ }^{\text {TM }}$ (MHOM/BR/75/M4147) positive control to perform relative quantification using the $2-\Delta \Delta \mathrm{Ct}$ method. ${ }^{10,11,16,17}$ If kmp11 was not detected, a pre-amplification step was performed as per the Perfecta Pre-Amp Supermix guidelines. In the case that $\mathrm{kmp} 11$ remained undetected after pre-amplification, the $18 \mathrm{~S}$ rRNA gene was used as a reference, and a relative quantification was performed using the $2-\Delta \Delta \mathrm{Ct}$ method. ${ }^{11-17}$ Relative LRV-1 copy number was calculated using the methods outlined by Zangger and colleagues, and further 
described in the following paragraphs. ${ }^{16}$ The "gold" standard source of LRV-1 in this analysis as well as the Zangger article is L. V. guyanensis. ${ }^{16}$ We acknowledge that there are inter-species differences in LRV-1 viral load; however, we do not have an LRV1 clone to calculate an absolute copy number. Moreover, by normalizing the relative abundance to the $L$. V. guyanensis $\mathrm{MHOM} / \mathrm{BR} / 75 / \mathrm{M} 4147$ strain, which is readily available from the ATCC, we are able to maintain consistent analysis across experiments and studies. Where copy number is recorded as N/A, this indicates an inability to calculate LRV-1 copy number because of a non-amplifiable kmp11 reference gene.

Data analysis. Descriptive statistics (proportions, mean with $\mathrm{SD}$, median, and range) were calculated for all variables. Differences between categorical variables were compared using Fisher's exact test or chi-squared analysis. Continuous variables were compared by Kruskal-Wallis test or Mann-Whitney $U$ test. Significance was set at $P<0.05$. Data were analyzed using GraphPad Prism (GraphPad, La Jolla, CA). Relative LRV-1 copy number was calculated using the $2-\Delta \Delta \mathrm{Ct}$ method, whereby the gold standard LRV-1-containing strain, L. (V.) guyanensis ATCC 50126 (MHOM/BR/75/M4147), was used as a positive reference control for each PCR run containing LRV1-positive $L$. (V.) braziliensis isolates. ${ }^{10,11,16,17}$

\section{RESULTS}

Clinical and demographic data. Of 208 specimens from patients with confirmed ATL, $78(38 \%)$ isolates were identified as $L$. (V.) braziliensis acquired in Peru, by local Peruvians $(n=76$, $97 \%$ ) or travelers to Peru $(n=2,3 \%)$ (Figure 1, Supplemental Table 1). One hundred thirty $(62 \%)$ patients were excluded because of acquisition of ATL outside of Peru and/or infection with a non-braziliensis species (Figure 1). Sixty-five (83\%) patients were male, whereas $13(17 \%)$ were female (Tables 1 and 2). Median age was 34 years (range $2-76$ years) (Tables 1 and 2 ). Thirty $(38.5 \%)$ isolates were derived from patients with LCL, whereas $26(33 \%)$ were from patients with inflammatory/ multifocal CL and 22 (28\%) were from patients with MCL/ML (Tables 1 and 2).

Clinical phenotype by demographics: Secondary outcomes. Median ages of patients were distributed across phenotypes as follows: 40.5 years (range 20-82 years) for those with MCL/ML, 31 years (range 13-68 years) for those with inflammatory/multifocal CL, and 31 years (range $2-76$ years) for those with $\operatorname{LCL}(P=0.72)$ (Table 1). No children or adolescents had an MCL/ML phenotype; those in the < 18 years age bracket manifested LCL $(n=4,50 \%)$ or inflammatory/multifocal CL ( $n=4,50 \%)$ exclusively (Table 3). Male gender $(n=65 / 78)$ was distributed across phenotypes as follows: $100 \%(n=22)$ with MCL/ML, 69\% $(n=18 / 26)$ with inflammatory/multifocal CL, and $83 \%(25 / 30)$ with LCL $(P=0.02)$ (Table 2). No females in the analysis had MCL/ML, whereas $31 \%$ and $17 \%$ of those with the inflammatory/multifocal and LCL phenotypes, respectively, were female (Table 2). To summarize the clinical phenotype by demographics data, MCL/ML was found exclusively in adult male enrollees.

LRV-1 prevalence by clinical phenotype: Primary outcome. A total of 21/78 (27\%) isolates contained LRV-1, whereas 57/78 (73\%) did not (Figure 1, Tables 1 and 2). Leishmania RNA virus 1 was detected in nine $(41 \%)$ isolates causing MCL/ML, five (19\%) isolates causing inflammatory/multifocal CL ulcers, and seven (23\%) isolates causing LCL, respectively $(P=0.21)$ (Table 2). Leishmania RNA virus 1 positivity was distributed across phenotypes as follows: $43 \%(9 / 21)$ of LRV-1-positive isolates were found in MCL/ML, 24\% (5/21) of LRV-1-positive isolates were found in inflammatory/multifocal ulcers, and $33 \%$ (7/21) of LRV-1-positive isolates were found in LCL $(P=0.19)$ (Table 3). However, LRV-1 positivity was detected in only one (10\%) isolate from patients $>60$ years $(n=10), 20$ $(33 \%)$ isolates from patients aged $19-59$ years $(n=60)$, and zero (0\%) isolates from patients $<18$ years $(n=8)(P=0.0591)$ (Table 3$)$.

Relative LRV-1 copy number (abundance). Relative LRV1 copy number (abundance) was calculated for 17/21 (81\%) isolates positive for LRV-1. Mean relative copy number of LRV-1 for isolates causing ML/MCL $(n=7)$ was $21.6 \pm 14.6$ copies (median 4.7, range $9.0 \times 10^{-2}-103.5$ copies), whereas for inflammatory/multifocal CL $(n=4)$, it was $5.5 \times 10^{-2} \pm 2.5 \times$ $10^{-2}$ copies (median $5.8 \times 10^{-2}$, range $2.9 \times 10^{-3}-1.0 \times 10^{-1}$ copies), and for LCL $(n=6)$, it was $8.3 \pm 4.1$ (median 7.4, range $8.5 \times 10^{-3}-27.2$ copies) $(P=0.11)$ (Figure $2 \mathrm{~A}$, Supplemental Table 1). Relative copy expression of LRV-1 in isolates causing $\mathrm{MCL} / \mathrm{ML}$ was higher than that in LRV-1-positive isolates causing inflammatory $\mathrm{CL}(P=0.02)$ (Figure $2 \mathrm{~B}$, Supplemental Table 1). There was no difference in relative copy expression of LRV-1 in isolates causing MCL/ML versus all CL $(P=0.30)$ and LCL $(P=0.94)$ (Figure 2A and B, Supplemental Table 1). To summarize, $\mathrm{LRV}-1$ relative copy number in $\mathrm{MCL} / \mathrm{ML}$ isolates was 392.5-fold higher than in isolates causing inflammatory/ multifocal CL.

\section{DISCUSSION}

Severity of ATL has been hypothesized to be associated with the viral endosymbiont LRV-1 for decades, with the first report of LRV-1 isolated from a human with cutaneous satellite lesions and lymphatic involvement after visiting Suriname. ${ }^{18}$ Since this initial report, there have been significant advancements and availability of molecular diagnostic tools to further

TABLE 1

Demographic data for 78 patients with Leishmania (Viannia) braziliensis isolates acquired in Peru by clinical phenotype

\begin{tabular}{|c|c|c|c|c|c|}
\hline Characteristic & Total, $N(\%)$ & $\begin{array}{l}\text { Mucocutaneous or mucosal } \\
\text { leishmaniasis }(n=22), N(\%)\end{array}$ & $\begin{array}{l}\text { Inflammatory/multifocal } \\
\mathrm{CL}(n=26), \mathrm{N}(\%)\end{array}$ & $\begin{array}{l}\text { Localized CL } \\
(n=30), N(\%)\end{array}$ & $P$-value \\
\hline Gender & & & & & 0.02 \\
\hline Male & $65(83)$ & $22(100)$ & $18(69)$ & $25(83)$ & \\
\hline Female & $13(17)$ & $0(0)$ & $8(31)$ & $5(17)$ & \\
\hline Median age, years (range) & $34(2-82)$ & $40.5(20-82)$ & $31(10-70)$ & $31(2-76)$ & 0.10 \\
\hline Leishmania RNA virus 1 status & & & & & 0.21 \\
\hline Positive & $21(27)$ & $9(41)$ & $5(19)$ & $7(23)$ & \\
\hline Negative & 57 (73) & $13(59)$ & $21(81)$ & $23(77)$ & \\
\hline
\end{tabular}

$\mathrm{CL}=$ cutaneous leishmaniasis 
TABLE 2

Demographic data for 78 patients with Leishmania (Viannia) braziliensis isolates acquired in Peru by LRV-1 status

\begin{tabular}{llcr}
\hline \multicolumn{1}{c}{ Characteristic } & Total, $N(\%)$ & LRV-1 positive $(n=21), N(\%)$ & LRV-1 negative $(n=57), N(\%)$ \\
\hline $\begin{array}{l}\text { Gender } \\
\text { Male }\end{array}$ & $65(83)$ & $19(91)$ & 0.50 \\
$\quad$ Female & $13(17)$ & $2(9)$ & $46(81)$ \\
$\begin{array}{l}\text { Median age, years (range) } \\
\text { Clinical phenotype }\end{array}$ & $34(2-82)$ & $29(20-68)$ & $11(19)$ \\
$\quad \begin{array}{l}\text { Mucocutaneous or mucosal } \\
\quad \text { leishmaniasis }\end{array}$ & $22(28)$ & $9(43)$ & $35.5(2-82)$ \\
$\quad$ Inflammatory/multifocal CL & $26(33)$ & $5(24)$ & $13(22)$ \\
$\quad$ Localized CL & $30(38.5)$ & $7(33)$ & $21(37)$ \\
\hline
\end{tabular}

investigate and understand the role of LRV-1 in ATL, and further accrual of data in humans. ${ }^{5,9,10,12,19-23}$ It has been shown that LRV-1 and Leishmania parasites have co-evolved with clustering of both the virus and the parasite in specific geographic locations. Given the species-specific and geographic correlates of observed phenotype in tegumentary leishmaniasis, LRV-1 has the potential to contribute to the diagnosis, treatment, and prognostic decision-making in the care of ATL patients. ${ }^{6}$ In this study, we examined the overall prevalence and possible correlation to clinical phenotypes of LRV-1 in clinical strains of $L$. (V.) braziliensis acquired locally and exported from Peru, a highly endemic country for $C L$ and MCL/ML. Our analysis reflects predominantly the specimens from patients both residing in and traveling to endemic areas of Peru and, thus, does not constitute a full survey of representative cases restricted to endemic highland and jungle areas of Peru, where the distribution of isolates and phenotypes may differ at a population level. Although we observed no direct relationship between LRV-1 positivity or negativity with three discrete phenotypes, we documented that patients manifesting MCL/ML had strains of $L$. $(V$.) braziliensis containing the highest relative copy numbers of LRV-1, a novel observation in this patient population.

We analyzed LRV-1 status in 78 isolates of $L$. (V.) braziliensis causing various clinical phenotypes of ATL from Peru and found an overall $27 \%$ prevalence, which is within the range reported previously from studies of strains in Latin America, specifically Peru. ${ }^{5,19-24}$ It has been shown that LRV-1 is not preferentially associated with a specific phenotype, ${ }^{20}$ although we herein determined that $41 \%$ of MCL/ML patients were LRV-1 positive, followed by LCL and inflammatory/ multifocal CL at $23 \%$ and $19 \%$, respectively. Unlike Cantanhêde et al., ${ }^{19}$ we noted no direct association of LRV-1 positivity prevalence with clinical phenotype; however, we documented an almost 400-fold higher relative LRV-1 copy number in isolates causing $\mathrm{MCL} / \mathrm{ML}$ than isolates causing inflammatory/multifocal CL, potentially supporting a possible LRV-1 association with mucosal disease, in particular. Our findings extend what was originally documented by Ives and colleagues in a murine model of ATL where it was observed that LRV-1 quantity was several-fold higher in metastasizing strains of $L$. (V.) guyanensis. ${ }^{7}$ The relationship of both LRV-1 prevalence and relative viral burden to clinical manifestations and observed phenotype warrant additional work in larger cohort of patients with ATL, specifically in patients with MCL/ML.

On average, LRV-1-positive isolates in this analysis originated from patients who were 6 years younger than those whose isolates were LRV-1 negative; however, those at the extremes of age in this analysis had very low rates of LRV-1 positivity. In addition, those with MCL/ML were an average of 8.5 years older than those with disease confined to the skin. No children or adolescents had either clinically manifest MCL/ ML or LRV-1-positive L. (V.) braziliensis isolates causing their disease. Given that progression to ML typically occurs many years after LCL, ${ }^{1,25}$ that patients with MCL/ML in this analysis were older is, in itself, unsurprising. One possible explanation for why LRV-1 may be less likely to occur in older patients who are from endemic settings is the recurrent, lifelong exposure, which could enable the parasite to harness the endogenous RNAi activity of the Viannia subgenus to eliminate the virus over time. ${ }^{26}$ In this study, all but one isolate from patients older than 60 years $(n=10)$ were found to be LRV- 1 negative, and no isolates from patients younger than 18 years were LRV-1 positive. Advanced age is associated with poorer T-cell response and a Th2-biased response, in particular, ${ }^{27}$ which in the case of ATL, is correlated to poorer immunologic control of infection and persistence of the amastigote in the phagolysosome. ${ }^{4}$ Similarly, the T-helper-1 (Th1)-to-T-helper-2 (Th2) ratio has been demonstrated to be the lowest in childhood and adolescence, with a peak during mid-adulthood and slight decline thereafter. ${ }^{28}$ Th2 predominance over Th1 is also an important factor in the progression to $\mathrm{ML} .^{29-32}$ Understanding the potential behavioral, and socioeconomic and biological underpinnings of the age distributions of LRV- 1 noted in this analysis will be, ultimately, important for accurate interpretation of the viral role in ATL pathogenesis.

TABLE 3

Leishmania RNA virus 1 (LRV-1) status and clinical phenotype according to age bracket in 78 patients with Leishmania (Viannia) braziliensis acquired in Peru

\begin{tabular}{lcrr}
\hline & & & Clinical phenotype \\
\cline { 2 - 3 } \multicolumn{1}{c}{ Age bracket } & $\begin{array}{c}\text { LRV-1 positivity } \\
(n=21), N(\%)\end{array}$ & $\begin{array}{c}\text { Mucocutaneous or mucosal } \\
\text { leishmaniasis }(n=22), N(\%)\end{array}$ & $\begin{array}{c}\text { Inflammatory/multifocal } \\
(n=26), N(\%)\end{array}$ \\
\hline$<18$ years $(n=8)$ & $0(0)$ & $0(0)$ & $4(50)$ \\
$19-59$ years $(n=60)$ & $20(33)$ & $18(30)$ & $18(30)$ \\
$>60$ years $(n=10)$ & $1(10)$ & $4(40)$ & $4(40)$ \\
\hline
\end{tabular}




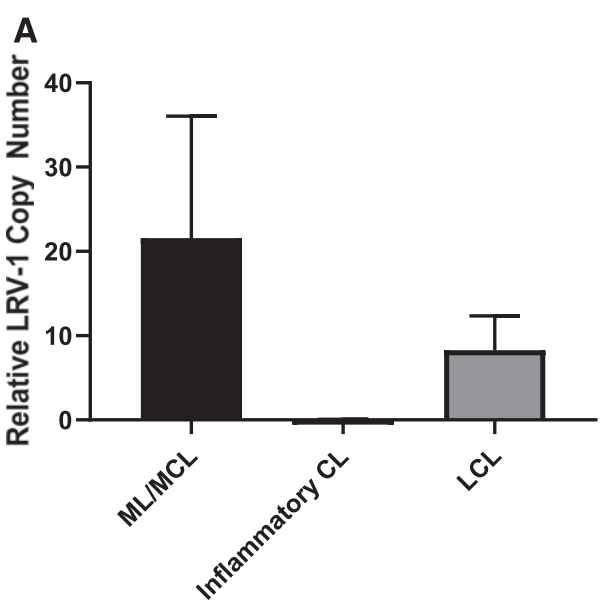

Clinical Phenotype

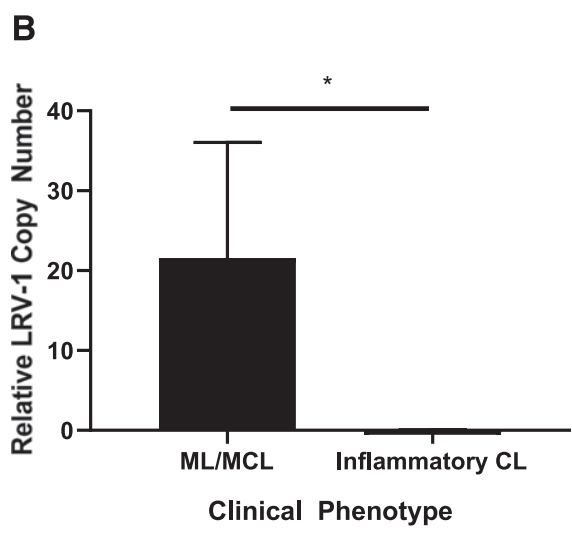

FIGURE 2. Mean relative Leishmania RNA virus 1 (LRV-1) copy number in isolates of Leishmania (Viannia) braziliensis by clinical phenotype of American tegumentary leishmaniasis compared by Kruskal-Wallis test (A). Mean relative LRV-1 copy number in isolates of $L$. (V.) braziliensis causing mucocutaneous or mucosal leishmaniasis (MCL/ML) and inflammatory/multifocal CL compared by Mann-Whitney test (B).

Limitations of this descriptive analysis of LRV-1 prevalence among $L$. (V.) braziliensis isolates originating from Peru include the comparatively small number of isolates from each age group (children, young adults, and older adults), which may have biased our interpretation of the data. Prospective enrolment of larger cohorts that might enable more even distribution of age brackets would be worthwhile. It is also possible that significantly different proportions of LRV-1 positivity by phenotype might have emerged with a larger cohort. Although our limited budget did not permit such a large-scale analysis, our findings are nevertheless important as, even in this smaller cohort, they document the higher relative viral load in $L$. $(V$.) braziliensis isolates causing MCL/ML and also suggest some interesting age preponderances that will be best interrogated using a combination of epidemiologic and basic scientific approaches going forward. Relative LRV-1 quantification in this study represents a relative copy number in relation to the ATCC $L$. (V.) guyanensis strain, where primary clinical samples are compared with a clonal line, which arguably has higher viral burden, given the oligoparasitic nature of clinical samples (which contain low amastigote burden, generally). All LRV-1-positive isolates in our analysis derive from a variety of primary clinical specimens, including cytology brushes, FPLIs, and a few cultured specimens. These isolates all reflect a mixed population and are not clonal lines; thus, our findings around relative LRV-1 copy number by strain should be interpreted cautiously. All estimates of relative LRV-1 copy number are based on methods that are highly dependent on the quality of procedures used to prepare samples and are based on a number of estimates. A truly accurate measure of LRV-1 copy number across strains will require development of improved methods. Another limitation of this analysis was our inability to resolve down to the final species-level Leishmania isolates from 20 individuals, some of whom may have been infected with $L$. $(V$.) braziliensis, which, again, may have influenced our findings. A prospective study following patients who are LRV-1 positive with CL over a significant time period and evaluating the likelihood of patients developing ML could shed light on the ability of LRV-1 to contribute to mucosal diseases while demonstrating the possibility of using antiviral therapy as a novel means of primarily or adjunctively treating patients.

\section{CONCLUSION}

We have demonstrated that relative LRV-1 viral burden was highest in $L$. (V.) braziliensis isolates causing mucosal involvement in this cohort of ATL acquired in Peru. Age emerged as an interesting bias in this cohort, where LRV-1-positive isolates originated from younger patients on average, but proportionate representation of LRV-1 positivity was not observed across age groups, with those within the extremes of age having low rates of LRV-1 positivity in their Leishmania isolates. Continued exploration of LRV-1 prevalence across age groups, particularly in larger cohorts, with specific interrogation of immunological age correlates of LRV-1 positivity while controlling for behavioral, socioeconomic, and other possible biological contributors to the age biases observed herein will be essential to understanding the relevance of this demographic variable to the host-parasite viral interplay that governs phenotype. The role of LRV-1 as a predictive biomarker of disease severity remains unclear; however, the mechanistic nature, particularly regarding the immune response, will prove useful to understanding overall ATL-LRV-1 pathogenesis, particularly in patients with MCL/ML.

Received February 18, 2019. Accepted for publication November 5, 2019.

Published online December 12, 2019.

Note: Supplemental table appears at www.ajtmh.org.

Financial support: This work was supported by Public Health Ontario via the Project Initiation Fund and the University of Toronto via an Early Career Department of Medicine award.

Authors' addresses: Ruwandi Kariyawasam, Institute of Medical Sciences, University of Toronto, Toronto, Canada, E-mail: ruwandi. kariyawasam@mail.utoronto.ca. Rachel Lau, Public Health Ontario Laboratory, Toronto, Canada, E-mail: rachel.lau@oahpp.ca. Braulio M. Valencia and Alejandro Llanos-Cuentas, Instituto de Medicina Tropical "Alejandro von Humboldt," Lima, Peru, E-mail: braulio. valencia@upch.pe and alejandro.llanos.c@upch.pe. Andrea K. Boggild, Tropical Disease Unit, Toronto General Hospital, Toronto, Canada, E-mail: andrea.boggild@utoronto.ca. 


\section{REFERENCES}

1. Reithinger R, Dujardin JC, Louzir H, Pirmez C, Alexander B, Brooker S, 2007. Cutaneous leishmaniasis. Lancet Infect Dis 7: 581-596.

2. Aronson N, Herwaldt BL, Libman B, Pearson R, Lopez-Velez R, Weina P, Carvalho EM, Ephros M, Jeronimo S, Magill A, 2016. Diagnosis and treatment of leishmaniasis: clinical practice guidelines by the Infectious Diseases Society of America (IDSA) and the American Society of Tropical Medicine and Hygiene (ASTMH). Clin Infect Dis 63: e202-e264.

3. Guimares LH et al., 2016. Atypical manifestations of cutaneous leishmaniasis in a region endemic for Leishmania braziliensis: clinical, immunological and parasitological aspects. PLoS Negl Trop Dis 10: e005100.

4. Hartlet MA, Ronet C, Zangger H, Beverley SM, Fasel N, 2012. Leishmania RNA virus: when the host pays the toll. Front Cell Infect Microbiol 2: 99.

5. Ginouves M, Simon S, Bourreau E, Lacoste V, Ronet C, Couppie P, Nacher M, Demar M, Prevot G, 2016. Prevalence and distribution of Leishmania RNA virus 1 in Leishmania parasites from French Guiana. Am J Trop Med Hyg 94: 102-106.

6. Catanhede LM, Fernandes FG, Ferreira GEM, Porrozzi R, Ferreira RGM, Cupolillo E, 2018. New insights into the genetic diversity of Leishmania RNA virus 1 and its species-specific relationship with Leishmania parasites. PLoS One 13: e0198727.

7. Ives A et al., 2011. Leishmania RNA virus controls the severity of mucocutaneous leishmaniasis. Science 331: 775-778.

8. Ronet C, Beverley SM, Fasel N, 2011. Muco-cutaneous leishmaniasis in the New World. Landes Biosci 2: 547-552.

9. Valencia BM, Adaui V, Chantry M, Alba M, Ramos A, Arevalo J, Llanos-Cuentas A, Boggild AK, 2014. Controversial role of Leishmania RNA virus as a determinant of pathogenicity in human leishmaniasis. Int J Infect Dis 21S: 165-166.

10. Ogg MM, Carrion R, de Carvalho Botelho AC, Mayrink W, CorreaOliveira R, Patterson JL, 2003. Quantification of Leishmania virus RNA in clinical samples and its possible role in pathogenesis. Am J Trop Med Hyg 69: 309-313.

11. Kariyawasam R, Grewal J, Lau R, Purssell A, Valencia BM, LlanosCuentas A, Boggild AK, 2017. Influence of Leishmania RNA virus 1 on proinflammatory biomarker expression in a human macrophage model of American tegumentary leishmaniasis. $J$ Infect Dis 216: 877-886.

12. Bourreau $E$ et al., 2016. Leishmania-RNA virus presence in L. guyanensis increases risk of first-line treatment failure and symptomatic relapse. J Infect Dis 213: 105-111.

13. Schonian G, Nasereddin A, Dinse N, Schweynoch C, Schallig HD, Presber W, Jaffe CL, 2003. PCR diagnosis and characterization of Leishmania in local and imported clinical samples. Diagn Microbiol Infect Dis 47: 349-358.

14. de Almeida ME, Steurer FJ, Koru O, Herwaldt BL, Pieniazek NJ, da Silva AJ, 2011. Identification of Leishmania spp. by molecular amplification and DNA sequencing analysis of a fragment of rRNA internal transcribed spacer 2. J Clin Microbiol 49: 3143-3149.

15. Wortmann G, Sweeney C, Houng HS, Aronson N, Stiteler J, Jackson J, Ockenhouse C, 2001. Rapid diagnosis of leishmaniasis by fluorogenic polymerase chain reaction. Am $J$ Trop Med Hyg 65: 583-587.

16. Zangger $\mathrm{H}$ et al., 2013. Detection of Leishmania RNA virus in Leishmania parasites. PLoS Negl Trop Dis 7: e2006.
17. Schmittgen TD, Livak KJ, 2008. Analyzing real-time PCR data by the comparative CT Method. Nat Protoc 3: 1101-1108.

18. Tarr PI, Aline RF, Smiley BL, Scholler J, Keithly J, Stuart K, 1988. LR1: a candidate RNA virus of Leishmania. Proc. Natl. Acad Sci USA 85: 9572-9575.

19. Cantanhede LM, Silva CF, Ito MM, Felipin KP, Nicolete R, Salcedo JMV, Porrozzi R, Cupolillo E, Ferreira RGM, 2015. Further evidence of an association between the presence of Leishmania RNA Virus 1 and the mucosal manifestations in tegumentary leishmaniasis patients. PLoS Negl Trop Dis 9: e0004079.

20. Adaui $V$ et al., 2016. Association of the endosymbiont doublestranded RNA virus LRV-1 with treatment failure for human leishmaniasis caused by Leishmania braziliensis in Peru and Bolivia. J Infect Dis 213: 112-121.

21. Macedo DH, Menezes-Neto A, Rugani JM, Rocha AC, Silva SO, Melo MN, Lye LF, Beverley SM, Gontijo C, Soares RP, 2016. Low frequency of LRV-1 in Leishmania braziliensis strains isolated from typical and atypical lesions in the State of Minas Gerais, Brazil. Mol Biochem Parasitol 210: 50-54.

22. Ito MM, Catanhede LM, Katsuragawa TH, Silva CP, Camargo LMA, Mattos RG, Vilallobos-Salcedo JM, 2015. Correlation between presence of Leishmania RNA virus 1 and clinical characteristics of nasal mucosal leishmaniosis. Braz $J$ Otorhinolaryngol 81: 533-540.

23. Pereira L, Maretti-Mira AC, Rodrigues KM, Lima RB, Oliveira-Neto M, Cupolillo E, Pirmez C, Oliveira MP, 2013. Severity of tegumentary leishmaniasis is not exclusively associated with Leishmania RNA virus 1 infection in Brazil. Mem Inst Oswaldo Cruz 108: 665-667.

24. Salinas G, Zamonra M, Stuart K, Saravia N, 1996. Leishmania RNA viruses in Leishmania of the Viannia subgenus. Am J Trop Med Hyg 54: 425-429.

25. Jara M, Valencia BM, Adaui V, Alba M, Lau R, Arevalo J, LlanosCuentas A, Boggild AK, 2016. Quantitative Kinetoplast DNA Assessment during treatment of mucosal leishmaniasis as a potential biomarker of Outcome: a pilot study. Am J Trop Med Hyg 94: 107-113.

26. Brettman EA et al., 2016. Tilting the balance between RNA interference and replication eradicates Leishmania RNA virus 1 and mitigates the inflammatory response. Proc Natl Acad Sci USA 113: 11998-12005.

27. Salam $N$ et al., 2013. T cell ageing: effects of age on development, survival \& function. Indian J Med Res 138: 595-608.

28. Chang W-S, Kim E-J, Lim Y-M, Yoon D, Son J-Y, Park J-W, Hong S-J, Cho S-H, Lee J-S, 2016. Age-related changes in immunological factors and their relevance in allergic disease development during childhood. Allergy Asthma Immunol Res 8: 338-345.

29. Moafi $\mathrm{M}$ et al., 2017. Comparison of pro-inflammatory cytokines of non-healing and healing cutaneous leishmaniasis. Scand $J$ Immunol 85: 291-299.

30. Tripathi $P$, Singh V, Naik S, 2007. Immune response to leishmanial: paradox rather than paradigm. FEMS Immunol Med Microbiol 51: 229-242.

31. Maspi N, Abdoli A, Ghaffarifar F, 2016. Pro- and anti-inflammatory cytokines in cutaneous leishmaniasis: a review. Pathog Glob Health 110: 247-260.

32. Hartley MA, Kohl K, Ronet C, Fasel N, 2013. The therapeutic potential of immune cross-talk in leishmaniasis. Clin Microbiol Infect 19: 119-130. 